\title{
Estudio comparativo del autoconcepto físico en adolescentes en función del género y del nivel de actividad físico-deportiva \\ Comparative study based on the physical self-concept in teenagers regarding gender and physical activity
}

\author{
*David Molero López-Barajas, *Francisca Ortega Álvarez, *Israel Valiente Martínez, *María Luisa Zagalaz Sánchez \\ *Universidad de Jaén (España)
}

\begin{abstract}
Resumen: Los propósitos del presente trabajo son evaluar el autoconcepto físico en la adolescencia, conocer los resultados obtenidos en las percepciones de las distintas dimensiones consideradas en el autoconcepto físico y comprobar la existencia de diferencias significativas en los resultados obtenidos en función de las variables género y nivel de práctica deportiva de los encuestados. La muestra está compuesta por 81 sujetos divididos en dos grupos: adolescentes estudiantes de secundaria obligatoria y nadadores en edad adolescente. Empleamos el Cuestionario de Autoconcepto Físico (CAF) como instrumento de recogida de información, el cual está compuesto por seis escalas: habilidad física, condición física, atractivo físico, fuerza, autoconcepto físico general y autoconcepto general. Ofrecemos los resultados obtenidos en dos análisis de la varianza, en el primero hemos encontrado diferencias significativas a nivel estadístico en las percepciones realizadas en función del género en las escalas habilidad física, condición física, fuerza y autoconcepto físico general $(\mathrm{p}<0,05)$, a favor de los hombres. En el segundo de los análisis, hemos comprobado la existencia de significatividad en las diferencias entre los dos grupos de adolescentes considerados en las escalas habilidad fisica y fuerza $(p<0,05)$, a favor de los que practican actividad física de manera habitual..
\end{abstract}

Palabra clave: deporte escolar, competición deportiva, educación.

\begin{abstract}
The aims of this article are to evaluate the physical self-concept in adolescence and to get to know the outcomes in the perceptions of the different dimensions in the physical self-concept. Furthermore, we aim to check the existence of noticeable differences in the outcomes regarding gender variables and regarding the level of physical activity of those polled. The sample consists of 81 individuals divided in two groups: secondary school teenager students and swimmers in adolescence. We use the Physical Self-concept Questionnaire (PSQ) as a tool for getting information; there are six scales: physical skills, physical conditions, physical charming, strength, and general physical self-concept. We use the Physical Self-concept Questionnaire (PSQ) as an instrument of collection of information which consists of 6 different scales: physical skills, physical condition, physical attractiveness, strength and general self-concept. We will show the results in two different analysis of the variant. In the first one we have found remarkable differences as far as the statistic point of view is concerned in gender perceptions in the scales of physical skills, physical condition, strength and general physical self-concept in favour of men $(\mathrm{p}<0,05)$. In the second analysis we have checked the existence of noticeable differences between the two groups of young people within the scales of physical skills and strength in favour of those who practice physical activity regularly $(\mathrm{p}<0,05)$. Key words: physical self-concept, gender, physical activity.
\end{abstract}

\section{Introducción}

El autoconcepto es uno de los aspectos más relevantes para el bienestar personal. De manera general, muchos aspectos conductuales se relacionan con esta variable. Pero si nos centramos en su relación con la práctica deportiva, se ha comprobado el efecto beneficioso que produce sobre él esta actividad en diversos estudios (Alferman y Stoll, 2000; Fox, 2000b; Moreno, Cervello y Moreno, 2008).

$\mathrm{El}$ autoconcepto es una variable que adquiere especial relevancia durante la adolescencia debido a los importantes cambios cognitivos, físicos y sociales que se producen en esta etapa (Cardenal y Fierro, 2003; Harter, 1999); además correlaciona positivamente con la calidad de vida (Huebner et al, 2004; Reiter y Golman, 1999) y con otras variables como el rendimiento académico (Gómez-Vela, Verdugo y González-Gil, 2007; Pons, 1998) o el efecto beneficioso de la actividad física sobre el autoconcepto en población adolescente (Fox, 1997, 2000a; Moreno, Cervello y Moreno, 2008), a pesar de éstos beneficios hay cada vez mayor número jóvenes sedentarios (García, 2005).

Lo que no admite ninguna duda es que en la sociedad actual se cultiva y se mima el cuerpo y la imagen corporal considerándolas como unas armas muy valiosas en las relaciones interpersonales. Ha quedado de manifiesto en diversos estudios (Harter, 1993; Fox, 2000b) la relación directa que existe entre el aumento de autocompetencia percibida y la aceptación personal, provocando una mejora de la autoestima, siendo el físico y la apariencia predictores de la autoestima (Fox, 1997).

\footnotetext{
Fecha recepción: 20-03-09 - Fecha envío revisores: 22-04-09 - Fecha de aceptación: 05-08-09 Correspondencia: David Molero López-Barajas

Facultad Humanidades y CEEE. Edificio D-2. Campus Las Lagunillas, s/n

Universidad de Jaén. 23071 - Jaén

E-mail: dmolero@ujaen.es
}

Los cambios evolutivos, en especial en la edad que nos ocupa, la adolescencia, parecen afectar directamente al autoconcepto físico, a pesar de que no existen muchos estudios longitudinales que permitan analizar su desarrollo a lo largo del tiempo. Según Núñez y GonzálezPineda (1994) el autoconcepto físico experimenta un descenso durante la preadolescencia (12 a 14 años) y una mayor diferenciación en entre sus componentes en la adolescencia. Otros autores (Goñi, Ruiz de Azúa y Rodríguez, 2004) no han encontrado diferencias significativas en función de la edad en adolescentes de 13 a 16 años, pero si han obtenido evidencias y diferencias significativas al realizar un análisis comparativo entre tres cohortes de edad $(<14$ años, 15-18 años y $>19$ años) utilizando como instrumento el Cuestionario de Autoconcepto Físico (Goñi, Ruiz de Azúa y Rodríguez, 2006), obteniéndose resultados en forma de U, es decir resultados más bajos en el grupo de edad intermedia. Garcia y Musitu (2001) en un amplio estudio para la baremación del AF5, analizaron el autoconcepto físico desde la adolescencia hasta la adultez, obteniendo resultados que ponen de manifiesto una disminución del mismo con la edad tanto para hombres como para mujeres.

La información presentada en nuestro estudio se obtuvo a través de las respuestas obtenidas con la aplicación del instrumento en los sujetos de la muestra durante el mes de octubre del año 2008. Como a continuación especificaremos, vamos a centrarnos en las diferencias existentes en la valoración del autoconcepto en función del nivel de la actividad físicadeportiva de los sujetos y del género. En relación a la primera de estas dos variables, se ha constatado los efectos positivos en la autoestima y de la práctica físico-deportiva en el autoconcepto físico (Bruya, 1977; Leith, 1994; Moreno, Cervello y Moreno, 2008), ya que a una mayor frecuencia de práctica de actividad física mejor es la salud mental del individuo disminuyendo la posibilidad de depresión (Kull, 2002) y en otros casos no sólo la depresión sino la ansiedad tanto en estado como 
en rasgo, incluso en población infantil y adolescente con necesidades educativas especiales (Gómez-Vela, Verdugo y González-Gil, 2007) o con cáncer (Bragado, Hernández-Lloreda, Sánchez-Bernados y Urbano, 2008).

En relación con el género, diversos estudios han demostrado que las mujeres tienen mayor preocupación por su cuerpo e imagen, demostrándose que éstas son más críticas con sus cuerpos y están más preocupadas por la imagen corporal que los hombres (Bane y Mc Auley, 1998; Loland, 1998). Las mujeres, a su vez, parecen vincular más el atractivo físico con el conjunto del autoconcepto, es decir con todos sus elementos, sin embargo los hombres se basan más en aspectos relacionados con la fuerza y el ego personal (Asçi, Asçi y Zorba, 1999; Hayes, Crocker y Kowalski, 1999).

Aún siendo numerosas las investigaciones realizadas en torno al autoconcepto, y en concreto en adolescentes, consideramos necesario abordar un estudio centrado en el autoconcepto físico en adolescentes adaptado a nuestro contexto, con los siguientes objetivos: (1) evaluar el autoconcepto físico durante la adolescencia, (2) conocer los resultados obtenidos en la valoración de las distintas dimensiones consideradas en el autoconcepto físico, y (3) comprobar si existen diferencias significativas a nivel estadístico en los resultados obtenidos en función de variables como el género y el nivel de práctica deportiva de los encuestados.

\section{Material y método}

\subsection{Sujetos}

La muestra del estudio responde a un muestreo no probabilístico de tipo accidental o casual «aquellos a los que el investigador tiene acceso» (Latorre, del Rincón y Arnal, 2003,p. 82) denominado por otros autores no aleatorio (Heinemann, 2003, p. 203). Los sujetos que componen la muestra son adolescentes $(\mathrm{n}=81)$ pertenecientes a dos grupos diferentes, uno de ellos estudiantes de Educación Secundaria con vida sedentaria (hecho verificado a través de la respuesta a una pregunta del cuestionario) y un grupo de adolescentes nadadores, todos ellos con edades comprendidas entre los 12 y los 18 años. La distribución de la muestra por género y por grupo aparece reflejada en la Tabla 1. El grupo de estudiantes de Educación Secundaria está compuesto por 59 sujetos, siendo 25 de ellos hombres y 34 mujeres; en el grupo de nadadores obtuvimos respuesta de 22 adolescentes siendo 11 hombres y 11 mujeres.

\begin{tabular}{|c|c|c|c|c|}
\hline \multicolumn{5}{|c|}{ Tabla 1. Distribución de la muestra $(\mathrm{n}=\mathbf{8 1})$} \\
\hline & & Estudiantes ESO & Nadadores & TOTAL \\
\hline \multirow[t]{2}{*}{$\mathrm{N}$} & & $59(72,8 \%)$ & $22(27,2 \%)$ & 81 \\
\hline & Rango & $14,0-16,0$ & $12,0-18,0$ & $12,0-18,0$ \\
\hline \multirow[t]{3}{*}{ Edad } & Media & 14,42 & 13,86 & 14,27 \\
\hline & Desv. Típica & 0,69 & 1,61 & 1,04 \\
\hline & Hombres & $25(30,86 \%)$ & $11(13,58 \%)$ & $36(44,4 \%)$ \\
\hline Género & Mujeres & $34(41,97 \%)$ & $11(13,58 \%)$ & $45(55,6)$ \\
\hline
\end{tabular}

\subsection{Instrumento}

Para obtener la información necesaria en esta investigación se ha utilizado el Cuestionario de Autoconcepto Físico-CAF-(Goñi, Ruiz de Azúa y Liberal, 2004; Goñi, Ruiz de Azúa y Rodríguez, 2006), prueba reconocida por la comunidad científica. El instrumento está compuesto por 36 ítems divididos en 6 dimensiones o subescalas [Habilidad Física (fiabilidadá=0.80), Condición Física (fiabilidadá=0.84), Atractivo Físico (fiabilidad á=0.88), Fuerza (fiabilidad á=0.83), Autoconcepto Fúsico
General (fiabilidadá=0.88) y Autoconcepto General (fiabilidadá=0.79)]; cada una de ellas está compuesta por 6 ítems.

\subsection{Método}

Esta investigación es de tipo no experimental y de carácter transversal, empleando una metodología que combina los estudios descriptivos y los de relación. Los análisis estadísticos realizados fueron llevados a cabo mediante el paquete estadístico SPSS 15.0. Se llevaron a cabo las pruebas para analizar los supuestos del análisis de varianza de normalidad, homogeneidad de las varianzas e independencia (Catena, Ramos y Trujillo, 2003, pp. 62-63); ejecutándose todos los procedimientos estadísticos con un nivel de confianza del 95\% (significación igual 0,05).

\subsection{Procedimiento}

El cuestionario fue aplicado de manera colectiva por los autores de este estudio a las dos cohortes analizadas en el mes de octubre del año 2008, después de obtener el consentimiento del centro educativo, así como el de las familias de los estudiantes y del club deportivo (los cuales pidieron la oportuna autorización a sus familiares) y recibir la colaboración de los mismos. En todos los casos, antes de la cumplimentación de los instrumentos se enfatizó el anonimato y la confidencialidad de los resultados, así como la voluntariedad de participar en el estudio, a su vez se explicaron las normas de contestación del mismo aclarando las dudas existentes. La muestra contestó a los cuestionarios en su aula habitual de clase (estudiantes de Educación Secundaria con vida sedentaria) y en su club deportivo (grupo de adolescentes nadadores), respectivamente. Los sujetos de la muestra desconocían el propósito del estudio, ciego único, con el fin conseguir sinceridad en las respuestas y reducir el efecto de la deseabilidad social, en la medida de lo posible. En el momento de la recogida de los cuestionarios se verifico la correctay completa contestación de los mismos. Una vez obtenidas las respuestas se procedió a la realización de los correspondientes análisis estadísticos.

\section{Resultados}

En la Tabla 2 se aprecian los estadísticos descriptivos obtenidos Media (en adelante M) y Desviación Típica (a partir de ahora DT) en cada de las dimensiones del instrumento para cada uno de los grupos considerados y para el total de la muestra. De manera global, para toda la muestra, la dimensión que obtuvo mayor valoración fueel Autoconcepto General $(\mathrm{M}=4,24)$ y el Autoconcepto Físico $(\mathrm{M}=4,09)$; en el grupo de Estudiantes de ESO también fueron estas dos dimensiones las que recibieron mayor valoración ( $\mathrm{M}=4,23$ y $\mathrm{M}=4,12$, respectivamente). Sin embargo, el grupo de adolescentes nadadores valoró en mayor medida el Autoconcepto General $(\mathrm{M}=4,25)$ y la Habilidad Física $(\mathrm{M}=4,02)$. Existió coincidencia, tanto para toda la muestra como para cada uno de los grupos, en la dimensión menos valorada siendo ésta la Fuerza en todos los casos (total muestra $\mathrm{M}=3,36$; Estudiantes $\mathrm{ESO}$ no deportistas $\mathrm{M}=3,25$ y Nadadores $\mathrm{M}=3,66$ ).

\begin{tabular}{|c|c|c|c|c|c|c|c|c|c|c|}
\hline \multirow{2}{*}{\multicolumn{2}{|c|}{ Variables }} & \multicolumn{3}{|c|}{ Estudiantes ESO } & \multicolumn{3}{|c|}{ Nadadores } & \multicolumn{3}{|c|}{ TOTAL } \\
\hline & & \multirow{2}{*}{\begin{tabular}{|r|} 
Varón \\
3,76
\end{tabular}} & \multirow{2}{*}{$\begin{array}{c}\text { Mujer } \\
3,44\end{array}$} & \multirow{2}{*}{$\begin{array}{r}\text { Total } \\
3,57\end{array}$} & \multirow{2}{*}{$\begin{array}{r}\text { Varón } \\
4,22\end{array}$} & \multirow{2}{*}{$\begin{array}{c}\text { Mujer } \\
3,81\end{array}$} & \multirow{2}{*}{$\begin{array}{c}\text { Total } \\
4,02\end{array}$} & \multirow{2}{*}{\begin{tabular}{|c|} 
Varón \\
3,90 \\
\end{tabular}} & \multirow{2}{*}{$\begin{array}{r}\text { Mujer } \\
3,53\end{array}$} & \multirow{2}{*}{$\begin{array}{r}\text { Total } \\
3,69\end{array}$} \\
\hline Habilidad & M & & & & & & & & & \\
\hline Física & DT & 0,86 & 0,75 & 0,80 & 0,57 & 0,68 & 0,65 & 0,80 & 0,74 & 0,79 \\
\hline \multirow{2}{*}{$\begin{array}{l}\text { Condición } \\
\text { Física }\end{array}$} & M & 3,72 & 3,28 & 3,47 & 3,80 & 3,50 & 3,65 & 3,75 & 3,33 & 3,52 \\
\hline & DT & 1,009 & 0,77 & 0,89 & 0,68 & 0,69 & 0,69 & 0,91 & 0,75 & 0,84 \\
\hline \multirow{2}{*}{$\begin{array}{l}\text { Atractivo } \\
\text { Físico }\end{array}$} & M & 4,06 & 3,72 & 3,87 & 3,84 & 3,63 & 3,74 & 4,00 & 3,70 & 3,83 \\
\hline & DT & 0,88 & 0,79 & 0,84 & 0,72 & 1,37 & 1,07 & 0,83 & 0,95 & 0,90 \\
\hline \multirow{2}{*}{ Fuerza } & M & 3,61 & 2,99 & 3,25 & 3,98 & 3,34 & 3,66 & 3,72 & 3,08 & 3,36 \\
\hline & DT & 0,94 & 0,63 & 0,83 & 0,59 & 0,72 & 0,72 & 0,86 & 0,66 & 0,82 \\
\hline \multirow{2}{*}{$\begin{array}{l}\text { Autoconcepto } \\
\text { Físico } \\
\text { General }\end{array}$} & M & 4,46 & 3,87 & 4,12 & 4,15 & 3,83 & 3,99 & 4,37 & 3,86 & 4,09 \\
\hline & DT & 0,77 & 0,70 & 0,78 & 0,60 & 1,19 & 0,94 & 0,73 & 0,83 & 0,82 \\
\hline \multirow{2}{*}{$\begin{array}{l}\text { Autoconcepto } \\
\text { General }\end{array}$} & $\mathbf{M}$ & 4,42 & 4,10 & 4,23 & 4,15 & 4,34 & 4,25 & 4,33 & 4,16 & 4,24 \\
\hline & DT & 0,73 & 0,68 & 0,71 & 0,64 & 0,603 & 0,61 & 0,71 & 0,66 & 0,68 \\
\hline
\end{tabular}




\begin{tabular}{|c|c|c|c|c|c|c|c|c|}
\hline \multicolumn{9}{|c|}{ Tabla 3. ANOVA: Género/subescalas del cuestionario CAF } \\
\hline Variables & Suma de cua & Irados & gl & $\begin{array}{c}\text { Media } \\
\text { cuadrática }\end{array}$ & $\mathbf{F}$ & Sig. & $\begin{array}{c}\text { Efecto } \\
\left(\eta^{2}\right)\end{array}$ & $\begin{array}{c}\text { Potencia } \\
\text { estadística }\end{array}$ \\
\hline \multirow{3}{*}{ Habilidad física } & Inter-grupos & 2,686 & 1 & 2,686 & 4,470 & 0,038 & 0,054 & 0,551 \\
\hline & Intra-grupos & 47,476 & 79 & 0,601 & & & & \\
\hline & Total & 50,162 & 80 & & & & & \\
\hline \multirow{3}{*}{$\begin{array}{r}\text { Condición } \\
\text { Física }\end{array}$} & Inter-grupos & 3,411 & 1 & 3,411 & 4,985 & 0,028 & 0,059 & 0,597 \\
\hline & Intra-grupos & 54,055 & 79 & 0,684 & & & & \\
\hline & Total & 57,466 & 80 & & & & & \\
\hline \multirow{3}{*}{ Atractivo Físico } & Inter-grupos & 1,767 & 1 & 1,767 & 2,168 & 0,145 & 0,027 & 0,307 \\
\hline & Intra-grupos & 64,395 & 79 & 0,815 & & & & \\
\hline & Total & 66,162 & 80 & & & & & \\
\hline \multirow{3}{*}{ Fuerza } & Inter-grupos & 8,368 & 1 & 8,368 & 14,522 & 0,000 & 0,155 & 0,964 \\
\hline & Intra-grupos & 45,525 & 79 & 0,576 & & & & \\
\hline & Total & 53,893 & 80 & & & & & \\
\hline \multirow{3}{*}{$\begin{array}{l}\text { Autoconcepto } \\
\text { Físico General }\end{array}$} & Inter-grupos & 5,074 & 1 & 5,074 & 8,047 & 0,006 & 0,092 & 0,80 \\
\hline & Intra-grupos & 49,815 & 79 & 0,631 & & & & \\
\hline & Total & 54,889 & 80 & & & & & \\
\hline \multirow{3}{*}{$\begin{array}{r}\text { Autoconcepto } \\
\text { General }\end{array}$} & Inter-grupos & 0,620 & 1 & 0,620 & 1,310 & 0,256 & 0,016 & 0,205 \\
\hline & Intra-grupos & 37,395 & 79 & 0,473 & & & & \\
\hline & Total & 38,015 & 80 & & & & & \\
\hline
\end{tabular}

Aparecieron diferencias estadísticamente significativas en 4 de las 6 subescalas del CAF en el análisis de la varianza (ANOVA) realizado en función del género de los encuestados (véase Tabla 3): Habilidad Física $\left[\mathrm{F}(1,79)=4,470 ; \mathrm{p}=0,038\right.$; Tamaño del efecto $\left(\mathrm{h}^{2}\right)=0,054$; Potencia estadística $=0,551(55,1 \%)]$; Condición Física $[\mathrm{F}(1,79)=4,985 ; \mathrm{p}=0,028$; Tamaño del efecto $\left(\mathrm{h}^{2}\right)=0,059$; Potencia estadística $\left.=0,597(59,7 \%)\right]$; Fuerza $\left[\mathrm{F}(1,79)=14,522 ; \mathrm{p}=0,000\right.$; Tamaño del efecto $\left(\mathrm{h}^{2}\right)=0,155$; Potencia estadística=0,964 (96,4\%)]; y Autoconcepto Físico General $\left[\mathrm{F}(1,79)=8,047 ; \mathrm{p}=0,006\right.$; Tamaño del efecto $\left(\mathrm{h}^{2}\right)=0,092$; Potencia estadística $=0,800(80,0 \%)]$. No se encontraron diferencias significativas en función del género en las subescalas Atractivo Físico $[\mathrm{F}(1,79)=2,168$; $\mathrm{p}=0,145$; Tamaño del efecto $\left(\mathrm{h}^{2}\right)=0,027$; Potencia estadística $=0,307$ $(30,7 \%)]$ y Autoconcepto General $[\mathrm{F}(1,79)=1,310 ; \mathrm{p}=0,256$; Tamaño del efecto $\left(h^{2}\right)=0,016$; Potencia estadística $\left.=0,205(20,5 \%)\right]$. Las diferencias significativas fueron favorables a los hombres en las 4 variables señaladas (Habilidad Física; Desviación Media [en adelante DM] $=0,37$, Condición Física $\mathrm{DM}=0,42$, Fuerza $\mathrm{DM}=0,64$ y Autoconcepto Físico General $\mathrm{DM}=0,51)$. No ha sido preciso realizar pruebas a posteriori o post-hoc al tener la variable independiente menos de tres grupos (hombre o mujer).

En el segundo ANOVA realizado se apreciaron diferencias estadísticamente significativas en 2 subescalas del $C A F$ en el análisis de la varianza realizado en función del nivel de actividad física de los adolescentes: Habilidad Física $[\mathrm{F}(1,79)=5,315 ; \mathrm{p}=0,024$; Tamaño del efecto $\left(h^{2}\right)=0,063$; Potencia estadística $\left.=0,624(66,24 \%)\right]$ y Fuerza $\left[\mathrm{F}(1,79)=4,172 ; \mathrm{p}=0,044\right.$; Tamaño del efecto $\left(\mathrm{h}^{2}\right)=0,050$; Potencia estadística $=0,523(52,3 \%)]$. Las diferencias significativas fueron favorables a los adolescentes deportistas frente a los que no lo son en las 2 dimensiones señaladas (Habilidad Física $\mathrm{DM}=0,42$ y Fuerza $\mathrm{DM}=0,45)$. Al igual que en el anterior ANOVA, no fue preciso realizar pruebas a posteriori o post-hoc al tener la variable independiente (grupo de adolescentes: estudiantes IES o Nadadores) menos de tres grupos.

\section{Discusión}

Algunos estudios previos que han analizado el autoconcepto en población adolescente (Fox, 2000a; García, 2005; Castro, Piéron y González, 2006; García, Musitu y Veiga, 2006; Esnaola, 2008; Moreno, Cervelloy Moreno, 2008) ofrecen resultados similares a los encontrados, al obtenerse significatividad en las diferencias en las subescalas de los componentes del autoconcepto físico como Habilidad Física, Condición Física, Fuerza y Autoconcepto Físico General, a favor de los hombres, sin embargo no se han constatado diferencias en el Atractivo Físico y en el Autoconcepto General entre hombres y mujeres. Estos datos demuestran la creciente presión social y preocupación que existe en la adolescencia sobre el atractivo físico por igual en los dos géneros, pero siguen siendo los chicos los más preocupados por los aspectos más vinculados con la práctica deportiva como habilidad, condición físicodeportiva, fuerza, etc.
En las valoraciones manifestadas por los adolescentes que no realizan práctica deportiva de manera habitual y aquellos otros que si la realizan, sólo hemos encontrado diferencias destacables significativamente en las subescalas Habilidad Física y Fuerza. Estos datos confirman que los adolescentes, de manera global, están preocupados por los aspectos relacionados con el atractivo y la apariencia física por igual, ya sean chicos o chicas, sedentarios o deportistas; sin embargo los adolescentes deportistas aportan valoraciones significativamente superiores en aquellas subescalas relacionadas directamente con aspectos vinculados con la práctica deportiva. Estas evidencias guardan relación con diversos estudios previos como los de Bruya (1977), Leith (1994), Moreno, Cervello y Moreno (2008), entre otros, en los que se trata la relación entre actividad física y autoconcepto físico; $y$ a su vez hay similitud con otros que analizan estas temáticas en muestras de adolescentes (GómezVela, Verdugo y González-Gil, 2007; Goñi, Ruiz de Azúa y Rodríguez, 2005).

\section{Conclusiones}

Se consideró oportuno ofrecer las conclusiones del presente trabajo tomando como referencia los objetivos planteados en el estudio. En relación al primero de ellos, evaluar el autoconcepto fisico durante la adolescencia, se aportaron evidencias en la muestra considerada en las distintas variables y dimensiones analizadas. En relación al segundo objetivo, conocer los resultados obtenidos en la valoración de las distintas dimensiones consideradas en el autoconcepto fisico, se ofreció información descriptiva diferenciada para cada uno de los grupos, para cada uno de los géneros y para el total de la muestra; analizando las dimensiones que han recibido la mayor valoración (Autoconcepto General y Autoconcepto Físico) y la menor valoración (Fuerza) por parte de la muestra, siendo analizadas a su vez, las dispersión de las puntuaciones obtenidas.

Finalmente, en relación al tercero de los objetivos, comprobar si existen diferencias significativas a nivel estadístico en los resultados obtenidos en función de variables como el género y el nivel de práctica deportiva de los encuestados, se encontraron diferencias significativas a nivel estadístico $(p<0,05)$ en función del género en las dimensiones Habilidad Física, Condición Física, Fuerza y Autoconcepto Físico General, a favor de los hombres. En relación el nivel de actividad física de los adolescentes, se constataron signifcatividad en las diferencias en las dimensiones Habilidad Física y Fuerza, a favor de los adolescentes que practican actividad física de manera habitual.

Aún siendo nuestros propósitos interesantes, no podemos generalizar los resultados ofrecidos ya que éstos se basan en una sola prueba y en un sólo momento de medida, motivos por los cuales tenemos que utilizar con cautela los mismos. Sin duda en futuros estudios sería de utilidad completar las evidencias aportadas con otras vías de evaluación y con estudios con muestras más amplias.

\section{Referencias Bibliográficas}

Alferman, D. y Stoll, O. (2000). Effects of physical exorcice on selfconcept and well-being. International Journal of Sport and Exercice Psychology, 30, 47-65.

Asçi, F.H. Asçi, A y Zorba, E. (1999). Cross-cultural validity and reliability of Physical self- Perception Profile. Journal of sport an exercise, 2, 1-11.

Bane, S. y McAuley, E. (1998). Body image and exercise. En J.L. Duda (Ed.). Advances in Sport an Exerxice Psysical Measuremente (pp. 311-322). Morgatonwn, WV: Fitness Information Technology.

Bragado, C., Hernández-Lloreda, Ma . J, Sánchez-Bernardos, Ma . L. y Urbano, S. (2008). Autoconcepto físico, ansiedad, depresión y autoestima en niños con cáncer y niños sanos sin historia de cáncer. Psicothema, 20 (3), 413-419.

Bruya, L.D. (1977). Effect of sleeted movement skills on positive selfconcept. Perceptual and Motor Skills, 45, 252-254. 


\begin{tabular}{|c|c|c|c|c|c|c|c|c|}
\hline & & & & Media & & & Efecto & Poter \\
\hline \multirow{4}{*}{$\begin{array}{r}\text { Habilidad } \\
\text { física }\end{array}$} & & & & & & & & \\
\hline & Inter-grupos & 3,162 & 1 & 3,162 & 5,315 & ,024 & 0,063 & 0,624 \\
\hline & Intra-grupos & 47,000 & 79 & ,595 & & & & \\
\hline & Total & 50,162 & 80 & & & & & \\
\hline \multirow{3}{*}{$\begin{array}{r}\text { Condición } \\
\text { Física }\end{array}$} & Inter-grupos &, 518 & 1 & ,518 & ,718 & ,399 & 0,009 & 0,133 \\
\hline & Intra-grupos & 56,948 & 79 &, 721 & & & & \\
\hline & Total & 57,466 & 80 & & & & & \\
\hline \multirow{3}{*}{$\begin{array}{r}\text { Atractivo } \\
\text { Físico }\end{array}$} & Inter-grupos & 263 & 1 &, 263 & ,316 & ,576 & 0,004 & 0,086 \\
\hline & Intra-grupos & 65,898 & 79 & 834 & & & & \\
\hline & Total & 66,162 & 80 & & & & & \\
\hline \multirow{3}{*}{ Fuerza } & Inter-grupos & 2,703 & 1 & 2,703 & 4,172 & ,044 & 0,050 & 0,523 \\
\hline & Intra-grupos & 51,190 & 79 & ,648 & & & & \\
\hline & Total & 53,893 & 80 & & & & & \\
\hline \multirow{3}{*}{$\begin{array}{l}\text { Autoconcepto } \\
\text { Físico General }\end{array}$} & Inter-grupos & ,291 & 1 & ,291 & ,421 &, 518 & 0,005 & 0,098 \\
\hline & Intra-grupos & 54,599 & 79 & 691 & & & & \\
\hline & Total & 54,889 & 80 & & & & & \\
\hline \multirow{3}{*}{$\begin{array}{r}\text { Autoconcepto } \\
\text { General }\end{array}$} & Inter-grupos & ,003 & 1 & ,003 & ,006 &, 938 & 0,000 & 0,051 \\
\hline & Intra-grupos & 38,012 & 79 & ,481 & & & & \\
\hline & Total & 38,015 & 80 & & & & & \\
\hline
\end{tabular}

Cardenal, V. y Fierro, A. (2003). Componentes y correlatos del autoconcepto en la escala de Piers-Harris. Estudios de psicología, 24 (1), 101-111.

Castro, Ma J., Piéron, M. y González, M. A. (2006). Actitudes y motivación en educación física escolar. Retos. Nuevas tendencias en Educación Física, Deporte y Recreación, 10, 5-22.

Catena, A., Ramos, M. M. y Trujillo, H. M. (2003). Análisis multivariado, un manual para investigadores. Madrid: Biblioteca Nueva.

Esnaola, I. (2008). El autoconcepto físico durante el ciclo vital. Anales de Psicología, 24 (1), 1-8,

Fox, K.R. (1997). The Physical self and processes in self-esteem development.En K.R. Fox (Ed.), The physical self: From motivation to well-being (pp. 111-139). Champaign, El: Human Kinetics.

Fox, K.R. (2000a). Self-esteem, self-perceptions and exercise. International Journal of Sport Psychology, 31, 288-240.

Fox, K.R. (2000b). The effects of exercise on self perceptions and selfesteem. En S.J.H. Biddle, K.R. Fox y S. H. Boutcher(eds.), Physical Activity and Psychological Well-Being (pp. 88-118). Londres: Routhledge.

García, M. (2005). Encuesta sobre hábitos deportivos de los españoles. Madrid: CIS.

Garcia, F. y Musitu, G. (2001). Autoconcepto Forma 5. AF5. Manual. Madrid: TEA.

García, J. F., Musitu, G. y Veiga, F. (2006). Autoconcepto en adultos en España y Portugal. Psicothema, 18 (3), 551-556.

Gómez-Vela, M., Verdugo, MA. y Gónzalez-Gil, F. (2007). Calidad de vida y autoconcepto en adolescentes con necesidades educativas especiales y sin ellas. Infancia y Aprendizaje, 30 (4), 499-522.

Goñi,A., Ruiz de Azúa, S. y Liberal, I. (2004). Propiedades psicométricas de un nuevo cuestionario para la medida del autoconcepto físico. Revista de Psicología del Deporte, 13 (2), 195-213.
Goñi, A. Ruiz de Azúa, S. y Rodríguez, A. (2004). Deporte y autoconcepto físico en la preadolescencia. Apunts, Educación Física $y$ Deportes, 77, 18-24

Goñi, A. Ruiz de Azúa, S. y Rodríguez, A. (2005). La importancia concedida a diversos aspectos del yo físico: un instrumento para su medida. Anales de Psicología, 21 (1), 92-101.

Goñi, A. Ruiz de Azúa, S. y Rodríguez, A. (2006). Cuestionario de Autoconcepto Físico (CAF). Manual. Madrid: EOS.

Harter, S. (1993). Causes and consequences of low self-esteem in children and adolescents. En R.B. Baumeister(Ed.), self-esteem: The puzzle of low self-esteem (pp. 57-78). Nueva York: Plenum Press.

Harter, S. (1999). The construction of the self. A developmental perspective. Nueva York: Guildford Press.

Hayes, S.D., Crocker P.R.E. y Kowalski K.C. (1999). Gender differences in physical self perceptions, global self-esteem, and physical activity: Evaluation of the Physical Self-Perception Profile model. Journal Sport, 22, 1-14.

Heinemann, K. (2003). Introducción a la metodología de la investigación empírica en Ciencias del Deporte. Barcelona: Paidotribo.

Huebner, E.S., Valois, R., Suldo, S., Smith, 1., McNight, C., Seligson, J. yZullig, K. (2004). Perceived quality of life:Aneglected component of adolescent health assessment and intervention. Journal of Adolescent Health, 34, 270-278.

Kull, M. (2002). The relationships between physical activity, health status and psychological well-being of fertiliyaged women. Scandinavian journal of Medicine \& Science in Sport, 12, 241-247.

Latorre, A., del Rincón, D. y Arnal, J. (2003). Bases metodológicas de la investigación educativa. Barcelona: Ediciones Experiencia.

Leith, L.M. (1994). Foundations of Exercise and Mental Health. Morgantown, WV: Fitness Information Technology.

Loland, N.W. (1998). Body image and physical activity. A survey among Norwegian men and women. International Journal of port and Exercise Psychology, 29, 339-365.

Moreno, J. A., Cervello, E. y Moreno, R. (2008). Importancia de la práctica físico-deportiva y del género en el autoconcepto físico de los 9 a los 23 años. International Journal of Clinical and Health Psychology, 8(1), 171-183.

Núñez, J. C. y González-Pineda, J. A. (1994). Determinantes del rendimiento académico. Variables cognitivo-motivacionales, atribucionales, uso de estrategias y autoconcepto. Oviedo: Universidad de Oviedo.

Pons, J. (1998). El autoconcepto en la infancia y adolescencia, y los agentes primarios de socialización. Información Psicológica, 66, 40-50.

Reiter, S. y Goldman, T. (1999). A programme for the enhancement of autonomy in young adults with physical disabilities: the development of a realistic self concept, individual perception of quality of life and the development of independent living skills. International Journal of Rehabilitation Research, 22, 71-74.
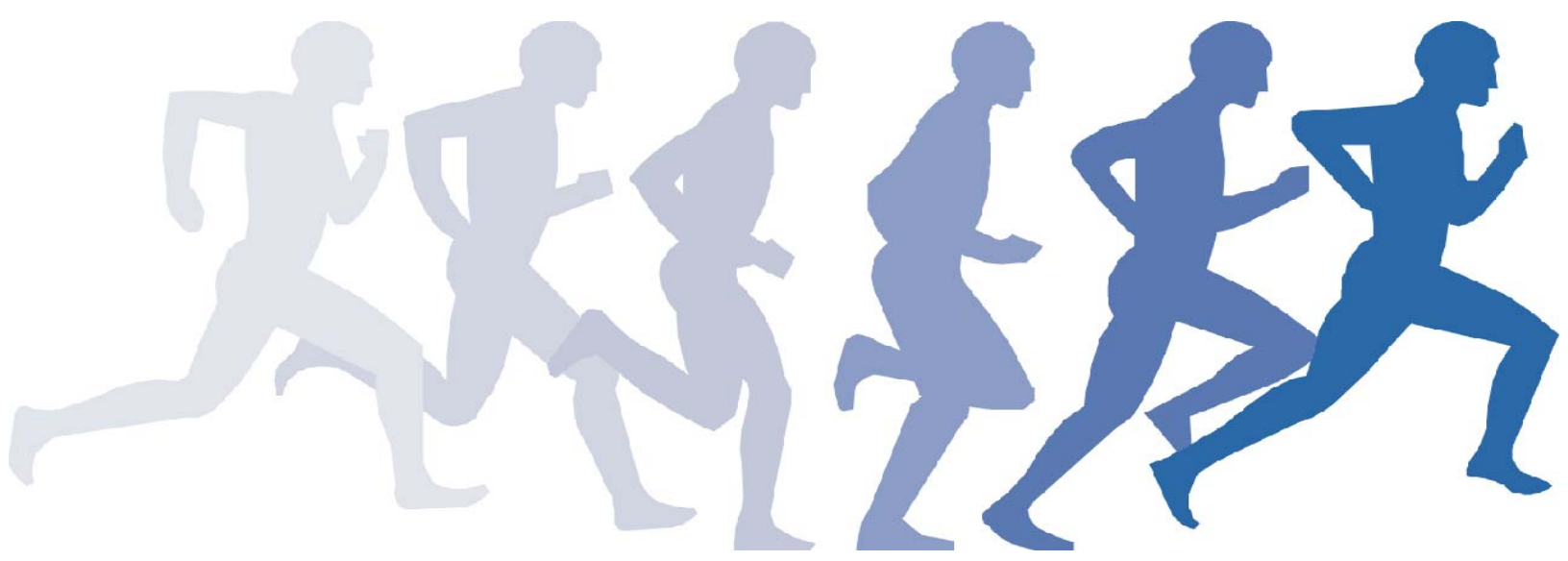